\title{
A Computational Algorithm for Path Interval Determination in Multi-Axis Filleted End Milling
}

\author{
Tsutomu Sekine ${ }^{1}$ \\ 1 Department of Systems Design Engineering, Faculty of Science and Technology, Seikei University, Tokyo, \\ Japan \\ e-mail: ts_s@outlook.com
}

\begin{abstract}
The demands for filleted end milling with high efficiency are increasing more and more. Path interval determination is one of the computational processes in the tool path generation, and its development can offer further improvement in multi-axis filleted end milling. However, to our knowledge, the practical approach has been hardly proposed so far. Hence, this study proposes a novel algorithm to determine a suitable path interval in multi-axis filleted end milling with a tool inclination. The procedure with wide applicability and robustness was described based on the 3D geometrical consideration of filleted end mill. Then, the characteristics of procedure were discussed with the visual data obtained from the computational results.
\end{abstract}

Keywords: tool path generation, path interval, multi-axis $\mathrm{CNC}$ machining, torus intersection problem, filleted end mill

\section{INTRODUCTION}

Mechanical machining is one of the core technologies in today's industry. Among the various processes, milling has been playing an especially important role as a versatile, highly-automated process. Computer numerical controlled (CNC) machine tools have widely contributed to advance manufacturing automation [1-3]. CNC machining technology gradually broadened its applicable fields with the development of CNC machine tools. Computer-aided technologies have also given impetus to the technological advancement. Computer-aided design (CAD) acted as one of the driving forces to create attractive products [4]. It is well known that a designed shape made in CAD has significant influence on product manufacturing. Computer-aided manufacturing (CAM) is a key technology for the modern production [5, 6]. A process plan implemented in CAM is an ultimate determinant of surface quality, machining accuracy, etc. in practical manufacturing. There still exist demands awaited highly such as higher machining speeds and longer tool life, and tool path generation has been studied for the further advancement of milling [7-10].

Although there are a lot of parameters determined in product manufacturing, path interval is known as one of the influential factors in tool path generation. Two path intervals have been considered to improve surface features after milling [11-13]. The one is a path interval along the feed direction of a tool, and the other is a path interval along the cross-feed direction of a tool. The most studies related to tool path generation have focused on the latter. Scallop height is also applied as a controllable factor to predict a path interval in these studies $[14,15]$. The formulas have been expressed based on geometrical relationships between path interval and scallop height in milling; furthermore, a few of them have contributed to achieving suitable balance between surface quality and machining efficiency [16]. There are several kinds of tool tip geometry in milling, so that the related works have been reported so far according to each of the shapes.

In ball end milling, a profile of tool tip geometry can be considered as a half circle projected 
onto an instantaneous section of designed shape [17]. The cross section is properly selected with tool movement. The geometrical consideration is similarly applicable even with a variation of tool inclination in milling [18]. A path interval formula is generally derived with the perspective after an intersection problem is set using two half circles obtained from adjacent tool paths. Solving the formula can lead to a suitable path interval or scallop height. There exist some formulas based on the geometrical consideration $[19,20]$.

On the other hand, different procedures are required in multi-axis flat and filleted end milling since these profiles projected onto an instantaneous section geometrically change with a variation of tool inclination [21-25]. The procedures with wide applicability, robustness, efficiency, and accuracy has been reported to determine a proper path interval in multi-axis flat end milling [26]. Meanwhile, the machining states in multiaxis filleted end milling can be classified into four general groups [27]. The two-dimensional (2D) consideration for tool tip geometry is questionable to express the geometrical relationships correctly. In the case of three-dimensional (3D) consideration, the intersection problems entail mathematical complexities in essence. One of the related studies proposed a novel procedure for a path interval determination with a reference point [28]. The findings indicated that the reference point was important to estimate a path interval correctly in the 3D consideration. However, little has been repotted on influences of geometrical relationship between a path interval and scallop height on a reference point [29]. In addition, the characteristics of reference point is not clear to apply it for path interval determination in filleted end milling.

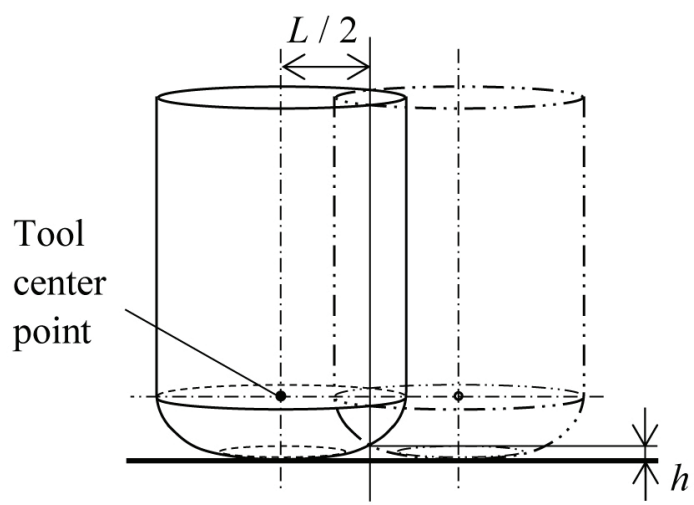

Fig. 1. A path interval $L / 2$ and scallop height $h$ in filleted end milling with a tool inclination along a tool feed direction
The purpose of this study is to provide a novel algorithm to determine a suitable path interval in multi-axis filleted end milling with a tool inclination. The procedure with wide applicability and robustness will be proposed based on the 3D geometrical consideration of filleted end mill. Then, the characteristics of procedure are discussed with the visual data obtained from the computational results.

\section{GEOMETRICAL EXPRESSION OF FILLETED END MILL}

This section describes geometrical expression to grasp typical features in filleted end milling. The expression with some parameters is based on the geometry of 3D space. Given that a machining state with a tool inclination along a tool feed direction, the milling on a plane can be modeled as a schematic illustration in Figure 1. Although a path interval usually indicates a distance between adjacent tool paths, we hereafter define $L / 2$ as a path interval, which is a unilateral distance from a tool center point to the controlled point of scallop height $h$. Let $R$ and $\rho$ be a tool radius and a tool inclination angle, as shown in Figure 2. We subsequently set several coordinate systems with reference to our previous works [e.g., 26]. Figure 3 shows a torus with some parameters to express geometrical features in the tool tip geometry of filleted end mill. In Figure 4, T coordinate system is introduced as a three-dimensional, orthogonal coordinate system with XT, YT, and ZT axis. The coordinate origin exists in the same position as a tool center point of filleted end mill. ZT axis also coincides with the tool rotational axis. In addition, $R_{b}$ and $R_{c r}$ are the major and minor radius of torus. The two radii have the following relationship: $R=R_{b}+R_{c r}$. Accordingly, the lower, outside part of torus is used in the following

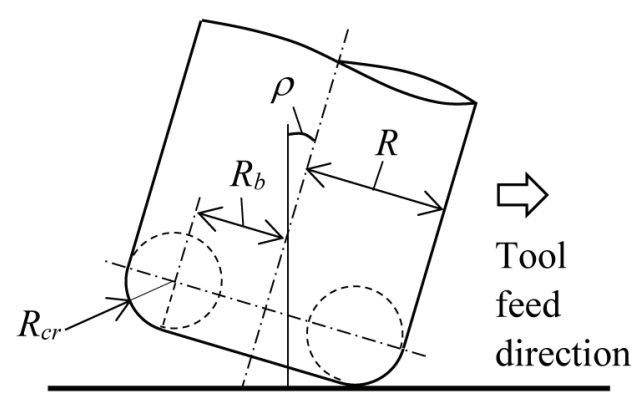

Fig. 2. Several radii and a tool inclination angle $\rho$ 


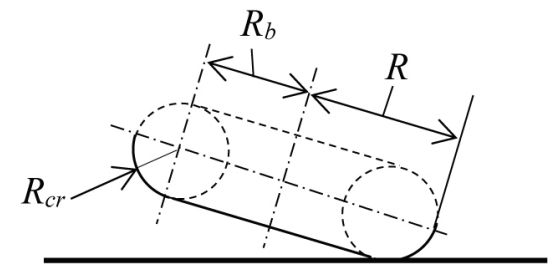

Fig. 3. A torus extracted to express a tool tip geometry of filleted end mill

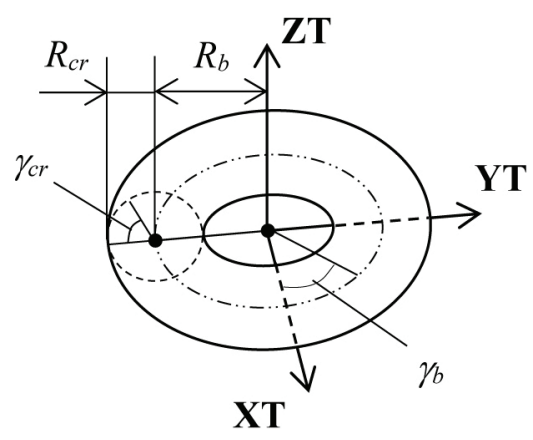

Fig. 4. $T$ coordinate system and some parameters related to a torus

computation dealing with the tool tip geometry of filleted end mill. Here, $\gamma_{b}$ is a parameter for the major radius of torus, and $\gamma_{c r}$ is a parameter for the minor radius of torus. The range of two parameters are from 0 to $2 \pi$. With use of the two parameters, an arbitrary point $\mathbf{P}_{\text {surf }}$ on a torus surface is given as follows:

$$
\mathbf{P}_{\text {surf }}=\left[\begin{array}{c}
R_{b} \cos \gamma_{b}+R_{c r} \cos \gamma_{c r} \cos \gamma_{b} \\
R_{b} \sin \gamma_{b}+R_{c r} \cos \gamma_{c r} \sin \gamma_{b} \\
R_{c r} \sin \gamma_{c r}
\end{array}\right]
$$

It is obvious that a path interval changes with variation of tool posture. After path intervals are individually calculated at key points in adjacent tool paths, the consideration of both sides based on uniting each path interval $L / 2$ enables us to estimate a practical pick feed distance under various tool postures.

\section{DERIVATION OF A SINGLE CONTACT POINT FOR PATH INTERVAL CALCULATION}

In this section, determination of a single contact point will be provided based on the geometrical expression described above. The initial value is indispensable for computing algorithm of path interval determination in filleted end milling.

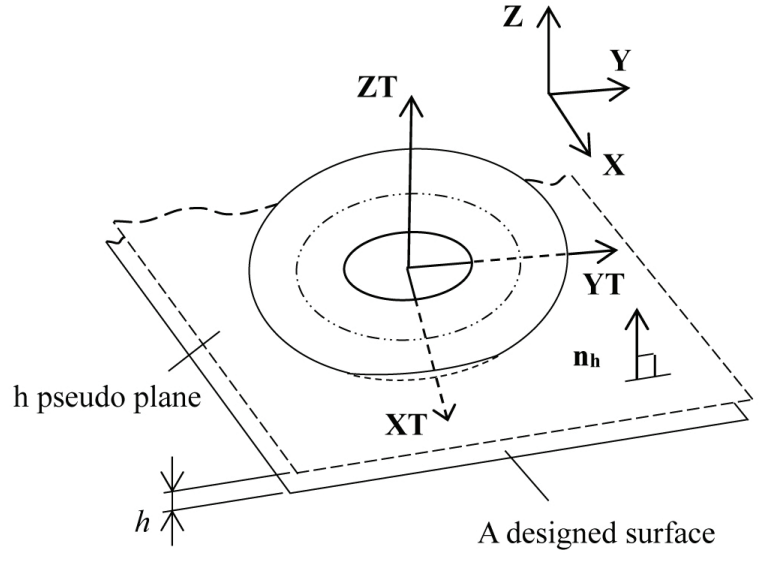

Fig. 5. A geometrical relationship between a torus and two planes

Figure 5 shows a relationship between a torus and two surfaces. In the figure, the bottom plane represents a surface of designed shape, and the upper plane is defined as an $h$ pseudo plane, which maintains a constant distance from a designed surface. The distance is identical with a scallop height $h$. Moreover, $\mathbf{n}_{\mathbf{h}}$ is the surface normal of $h$ pseudo plane. In Figure 5, X, Y, and $\mathbf{Z}$ axis constitute global coordinate system. The components of each axis are set as $(1,0,0),(0,1$, $0)$, and $(0,0,1)$, respectively. The direction of Y Y axis is the same as that of $\mathbf{Y}$ axis, and a tool feed is the direction of $\mathbf{X}$ axis. When the torus has an inclination along a tool feed direction, an angle between $\mathbf{X T}$ and $\mathbf{X}$ axis can accordingly give the tool inclination angle $\rho$.

As shown in Figure 6, we subsequently consider the case that the torus's section always emerges as a perfect circle even with variation of the cutting part. Then, each center point of the sections inevitably locates on the perimeter of a circle with $R_{b}$. Each section can be geometrically obtained using the center point's position and the inclination of section. There exist three positional relations regarding an intersection of the section and $h$ pseudo plane. The two patterns are a line of intersection and single contact. The other is without the intersection and contact. Here, a single contact is uniquely determined when a torus geometrically takes an arbitrary posture. This essence enables us to get a single contact point for path interval determination in filleted end milling.

For the following discussion, $\mathbf{P}_{\text {scp }}$ is used to indicate the center point achieving a single contact with $h$ pseudo plane. Notation with subscript scp expresses the calculation results related directly to $\mathbf{P}_{\text {scp }} . \mathbf{P}_{\text {base }}$ is set as a position vector when 


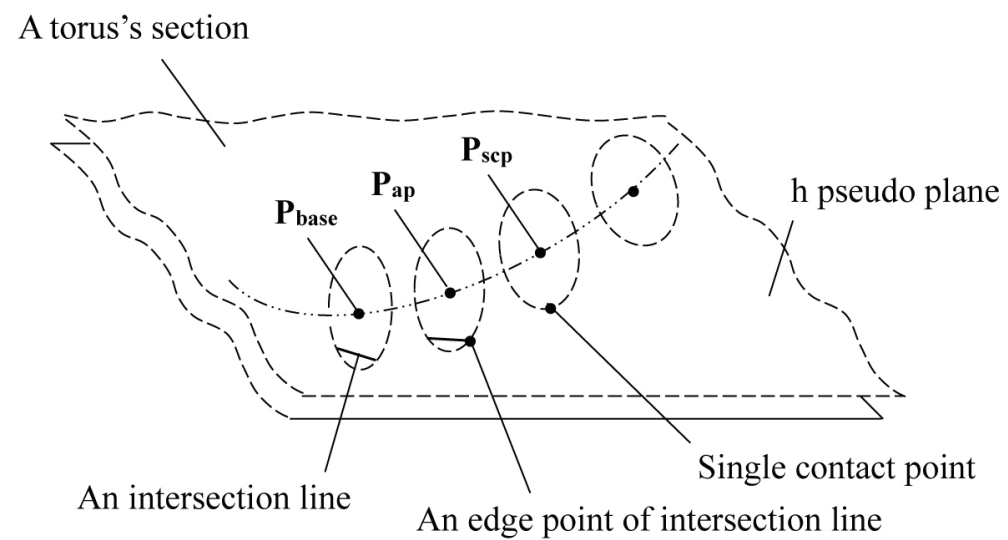

Fig. 6. Single contact point and intersection lines between torus's sections and h pseudo plane

$\gamma_{b}=0$. The section at $\mathbf{P}_{\text {base }}$ coincides with $\mathbf{X T}$ axis. In addition, let $\mathbf{t}_{\text {base }}$ be the tangent vector at $\mathbf{P}_{\text {base }}$. On the other hand, we set a search range on a circle with $R_{b} . \mathbf{P}_{\text {start }}$ and $\mathbf{P}_{\text {end }}$ are the start and end point of search range, respectively. $\mathbf{P}_{\text {end }}$ is commonly set as a position vector when $\gamma_{b}=0.5 \pi$. From these definitions, a single contact arises between a designed shape and the torus's section at $\mathbf{P}_{\text {base }}$. In this case, the distance between $\mathbf{P}_{\text {base }}$ and a designed shape is completely equal to $R_{c r}$, which indicates the miner radius of torus. The shortest distance from $\mathrm{h}$ pseudo plane is simultaneously obtained on this section. $\mathbf{P}_{\text {ap }}$ is used at an arbitrary position between $\mathbf{P}_{\text {start }}$ and $\mathbf{P}_{\text {end }}$. The position vectors in these sections can be easily determined through rotating $\mathbf{P}_{\text {base }}$ around $\mathbf{Z T}$ axis. Moreover, a tangent vector $\mathbf{t}_{\text {ap }}$ at $\mathbf{P}_{\text {ap }}$ can be also calculated in the same manner, as shown in Figure $7 . \mathbf{m}_{\text {ap }}=$ $\mathbf{t}_{\text {ap }} \times \mathbf{n}_{\mathbf{h}}$ since $\mathbf{t}_{\mathrm{ap}}$ is the tangent vector at $\mathbf{P}_{\mathrm{ap}} . \stackrel{\mathrm{d}}{\mathrm{ap}}_{s}$ is a distance between a section's center point and $\mathrm{h}$ pseudo plane, and it is along an inclination of torus's section. For the calculation, $d_{v}$ can be used as a vertical distance between $\mathbf{P}_{\text {ap }}$ and $\mathrm{h}$ pseudo plane. It is noted that the variation of $d_{s}$ is exactly associated with the directional variation of $\mathbf{t}_{\text {ap }}$ at each center point of sections. $\mathbf{P}_{\mathrm{hp}}$ can be calculated from these geometrical relationships, so that $d_{s}=\left|\mathbf{P}_{\mathrm{ap}}-\mathbf{P}_{\mathrm{hp}}\right|$. It is geometrically obvious that $d_{s}$ changes with variation of section.

The calculation of $d_{s}$ at each center point of sections can be given as follows:

1. Calculate $\mathbf{P}_{\text {ap }}$ and $\mathbf{t}_{\text {ap }}$ in torus's section through rotating $\mathbf{P}_{\text {base }}$ and $\mathbf{t}_{\text {base }}$ around $\mathbf{Z T}$ axis, with a rotational angle of $\gamma_{b}$.

2. Calculate the direction cosine $\eta$ between $\mathbf{t}_{\mathrm{ap}}$ and $\mathrm{h}$ pseudo plane, with the following equation:

$$
\eta=\mathbf{t}_{\text {ap }} \cdot\left\{\left(\mathbf{n}_{\mathbf{h}} \times \mathbf{t}_{\text {ap }}\right) \times \mathbf{n}_{\mathbf{h}}\right\} .
$$

3. Calculate $d_{s}$, which is the distance between $\mathbf{P}_{\text {ap }}$ and $\mathbf{P}_{\mathrm{hp}}$, as follows:

$$
d_{s}=\frac{\left(R_{c r}-h+R_{b} \sin \rho-R_{b} \cos \psi \sin \rho\right)}{\eta} .
$$

The above procedure can search $d_{s}$ corresponding to $R_{c r}$. Then, the torus's section and $\mathrm{h}$ pseudo plane have a single point contact. In addition, the center point becomes one of the reference points to determine path interval $L / 2$ for pre-determined scallop height $h$.

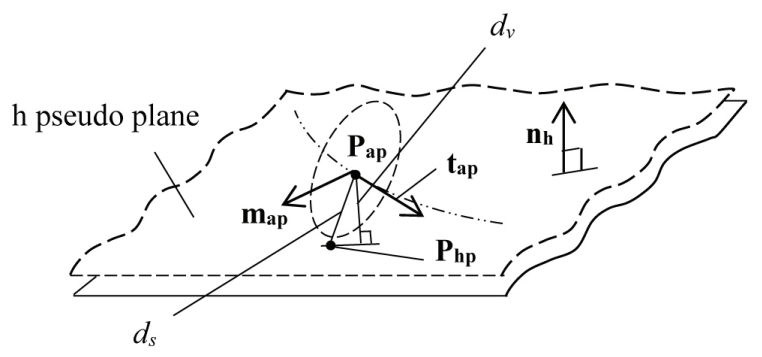

Fig. 7. Geometrical relationships in some parameters related to a torus's section

\section{COMPUTATIONAL ALGORITHM OF PATH INTERVAL DETERMINATION}

This section provides a novel algorithm to compute a suitable path interval in filleted end milling. After $\mathbf{P}_{\text {base }}$ is given on a circle with $R_{b}$, the search range to find the maximum value of $L / 2$ is firstly set as a partial curve from $\mathbf{P}_{\text {start }}$ to $\mathbf{P}_{\text {end }}$. Then, path interval determination is implemented to achieve the maximum value. When $\varepsilon$ is the convergence condition of iterative calculation, the procedure is as follows:

1) set an initial value of $\gamma_{b}$ using the following formula: $R_{b} \sin \rho-R_{b} \cos \gamma_{\beta} \sin \rho=0.5 h$.

2) search $\mathbf{P}_{\text {scp }}$ with updating the value of $\gamma_{b}$ when $\mid$ 
$R_{c r}-d_{s} \mid<\varepsilon$, where the updating is implemented with reference to the value of $R_{c r}-d_{s}$.

3) compute $\mathbf{m}_{\text {scp }}=\mathbf{t}_{\text {scp }} \times \mathbf{n}_{\mathrm{h}}$, and calculate $\mathbf{u}_{\text {scp }}$ through rotating $\mathbf{t}_{\text {scp }}$ around $\mathbf{m}_{\text {scp }}$ axis, with the rotational angle of $0.5 \pi$.

4) get $\mathbf{P}_{\text {hp }}$ through moving $\mathbf{P}_{\text {scp }}$ in the direction of $\mathbf{u}_{\text {scp }}$ and by the magnitude of $R_{c r}$, where $\mathbf{P}_{\mathrm{hp}}$ and $\gamma_{b}$ at $\mathbf{P}_{\text {scp }}$ become the start point of search range in computing algorithm. For the simplicity, $\gamma_{b s c p}$ is used at this point.

5) determine a search range after calculating $\gamma_{b}$ as the end point of search range in computing algorithm, where the calculation is implemented so as to have the similar Y-axis components at two kinds of $\mathbf{P}_{\mathrm{hp}}$.

6) divide the search range from $\gamma_{b}$ to $\gamma_{b s c p}$ into proper even numbers, and calculate $\mathbf{P}_{\mathbf{h p}}$ at each point.

7) find $\mathbf{P}_{h p}$ with the maximum of Y-axis component, and replace it to be the center of search range, which is gradually shrank. In addition, $\gamma_{b}$ and $\gamma_{b s c p}$ are updated for the next calculation after shrinking the search range.

8) repeat the step 6 and 7 until the difference of Y-axis component at adjacent $\mathbf{P}_{\mathrm{hp}}$ calculated in the step 6 is smaller than $\varepsilon$. From the iterative computation, a suitable path interval $L / 2$ can be finally acquired at $\mathbf{P}_{\mathrm{hp}}$ with the maximum of Y-axis component.

Figure 8 shows the pseudo-code of the above algorithm. Using the above algorithm, we can definitely obtain a suitable path interval $L / 2$ without divergence. Moreover, the algorithm can cover the variation of tool posture with the use of coordinate transformation.

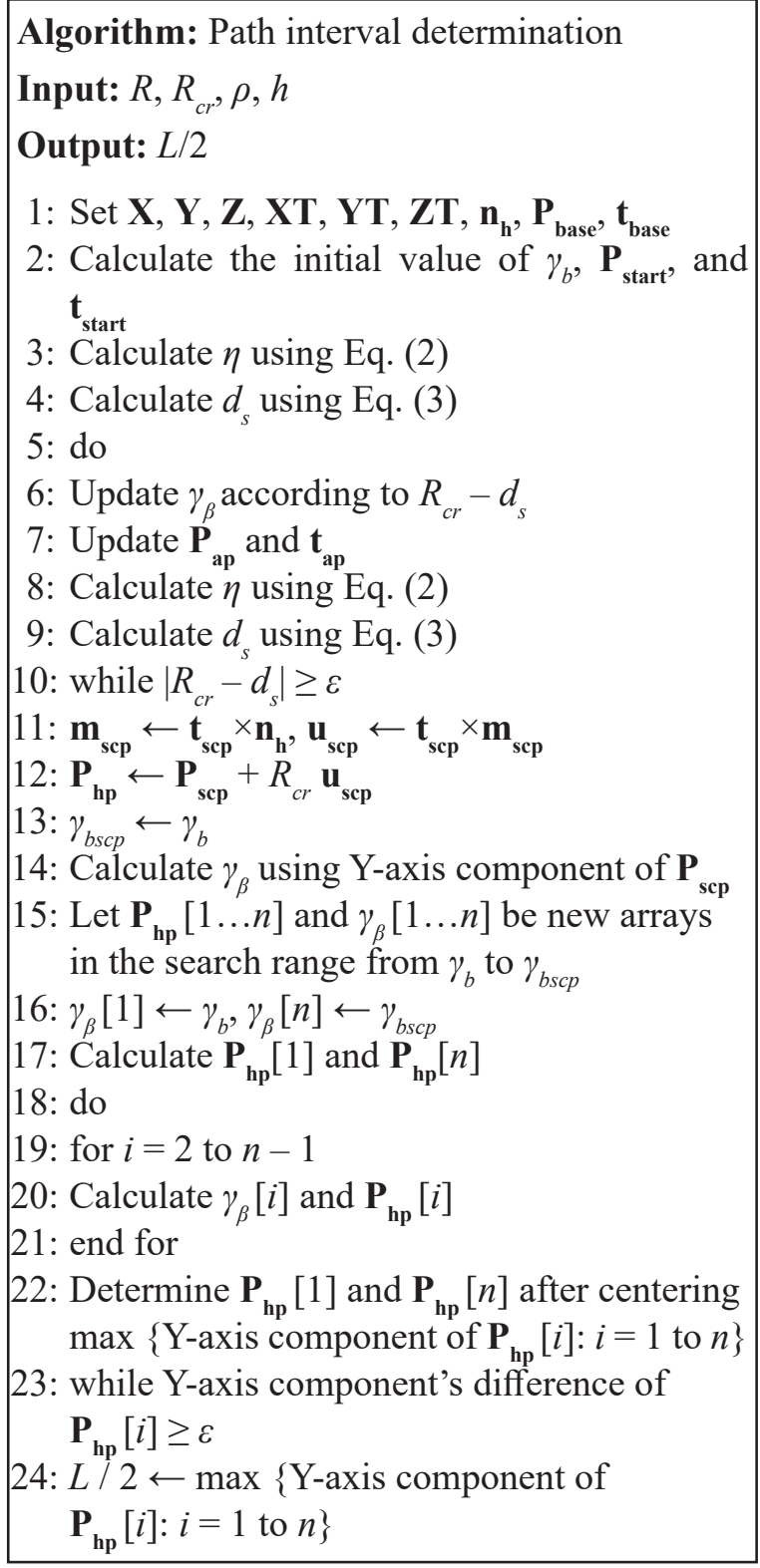

Fig. 8. Computational algorithm for path interval determination

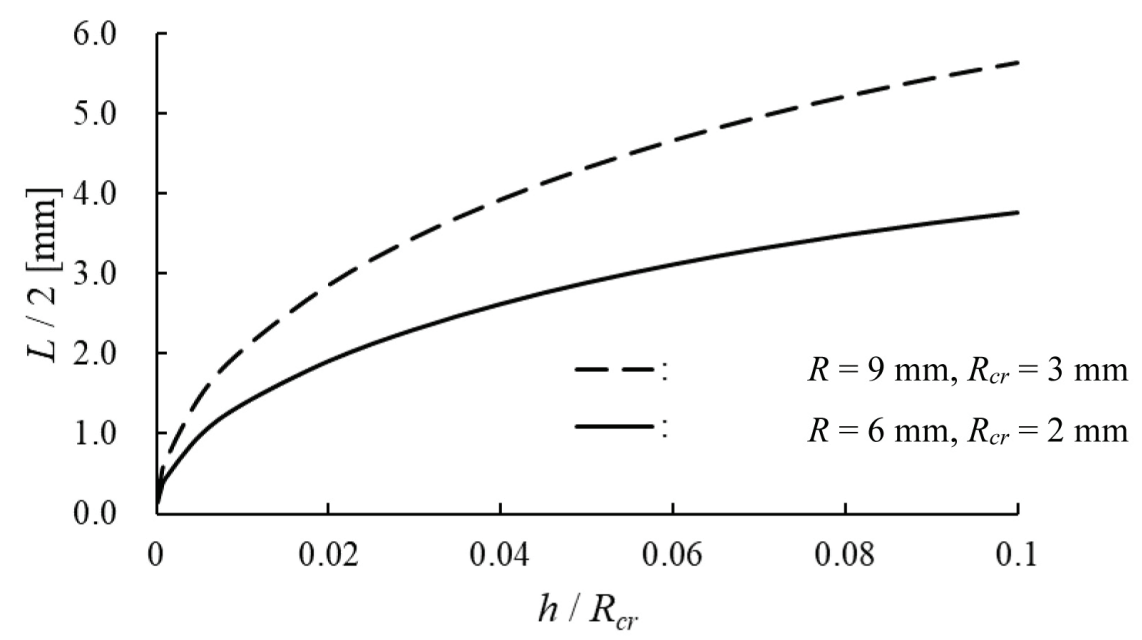

Fig. 9. Variations of $L / 2$ with $h / R_{c r}$ when $\rho=5.0 \mathrm{deg}$. 


\section{CHARACTERISTICS OF COMPUTATIONAL} ALGORITHM AND DISCUSSION

This section describes some characteristics of computational algorithm explained above. Figure 9 indicates the relationships between $h / R_{c r}$ and $L / 2$ in the case that $\rho=5 \mathrm{deg}$. Similarly, those for $\rho=10$ deg. are displayed in Figure 10. Two types of filleted end mills were used to create these graphs. The one was $R=9 \mathrm{~mm}$ and $R_{c r}=3$ $\mathrm{mm}$, and the other was $R=6 \mathrm{~mm}$ and $R_{c r}=2 \mathrm{~mm}$. Each tool was $R_{c r} / R=1 / 3$. It was found from each graph that the path interval $L / 2$ increased with increasing $R$. In addition, $L / 2$ rose with increasing $h / R_{c r}$. Especially, there was a considerable rise in the small range of $h / R_{c r}$. The difference of $L / 2$ between both tools was almost 1.5 times in the range from 0 to 0.1 of $h / R_{c r}$. The ratio was equal to the value obtained through dividing the tool with $R$ $=9 \mathrm{~mm}$ by the tool with $R=6 \mathrm{~mm}$. The similar ratio could be observed in Figure 10. Although iterative calculations were implemented in the computational algorithm, the similar ratio existed in the different conditions. The findings was extremely useful to estimate a path interval in filleted end milling. From the comparison of two graphs, it was obvious that $L / 2$ became large in any case when $\rho=5 \mathrm{deg}$.

Figure 11 shows the relationships between $h / R_{c r}$ and $L / 2$ in the case that $\rho=5 \mathrm{deg}$, while three types of filleted end mills were used to make the graph. Two comparisons were demonstrated in the figure. Namely, the one aims to investigate the influence of $R_{b}$, and the other is intended to reveal the effect of $R_{c r}$. In the former, two types of tools were $R_{b}=4 \mathrm{~mm}(R=6 \mathrm{~mm})$ and $R_{b}=6$ $\mathrm{mm}(R=8 \mathrm{~mm})$. These tools had the same size of $R_{c r}$. The computational results showed that $L / 2$ increased with increasing $R_{b}$. In the latter, two types of tools were $R_{c r}=1 \mathrm{~mm}(R=7 \mathrm{~mm})$ and $R_{b}=2$ $\mathrm{mm}(R=8 \mathrm{~mm})$. These tools had the same size of $R_{b}$. It was found from the comparison that $L / 2$

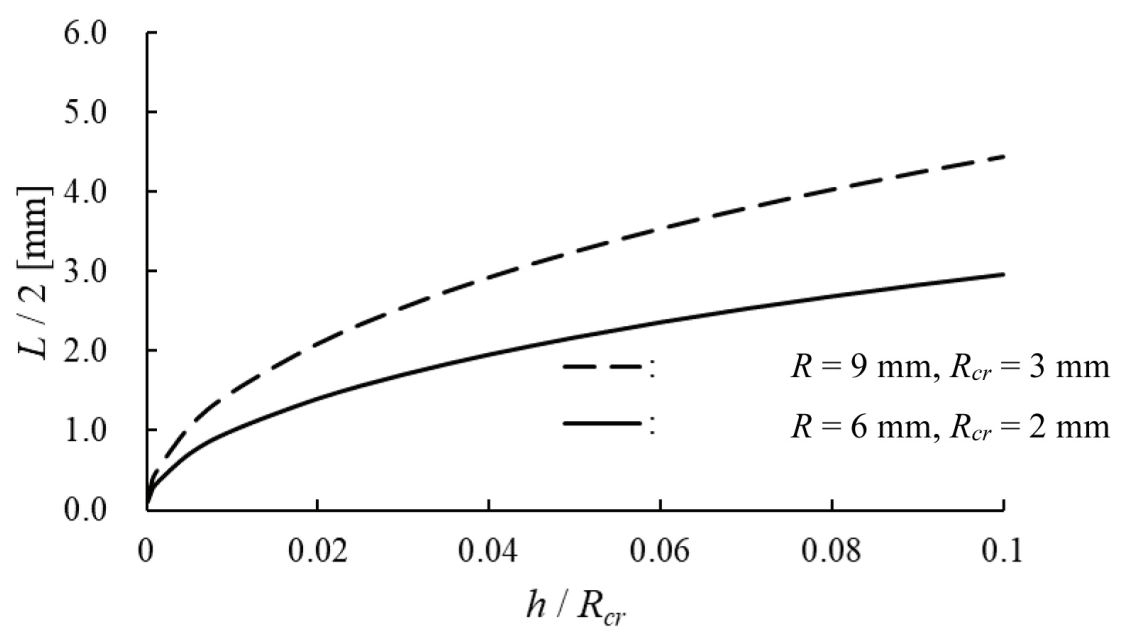

Fig. 10. Variations of $L / 2$ with $h / R_{c r}$ when $\rho=10.0 \mathrm{deg}$.

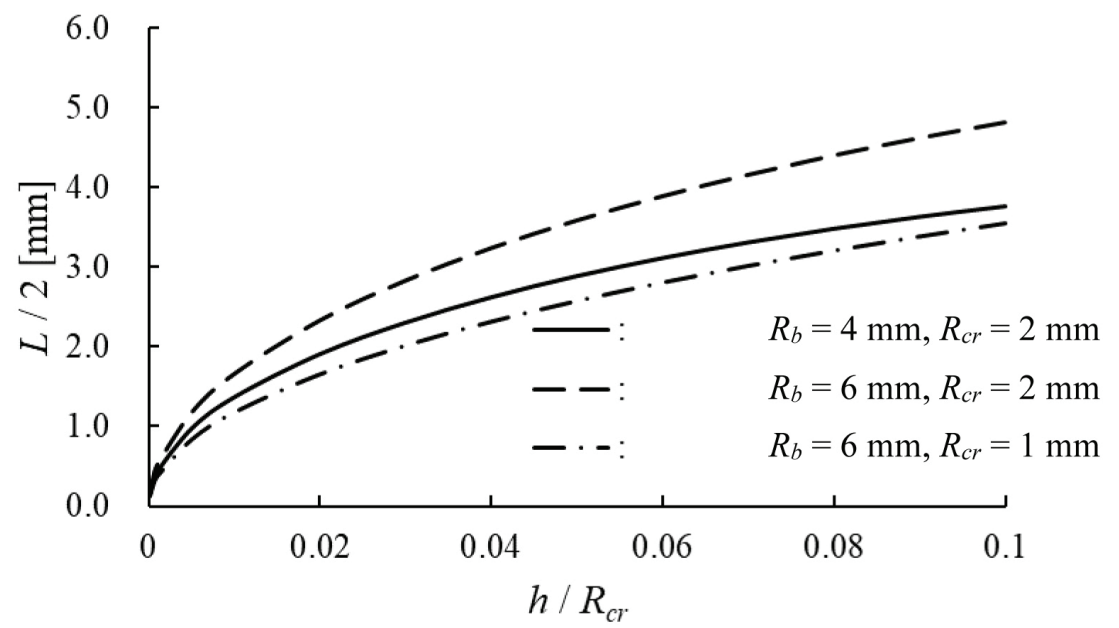

Fig. 11. Variations of $L / 2$ with $h / R_{c r}$ for analyzing the influence of $R_{b}$ and $R_{c r}$ when $\rho=5.0 \mathrm{deg}$. 
increased with increasing $R_{c r}$. From these results, the influence of $R_{c r}$ on the variation of $L / 2$ is larger than that of $R_{b}$. This fact is critically important to determine a suitable path interval in filleted end milling, so that it should be adequately careful about various situations with variation of $R_{c r}$.

\section{CONCLUSIONS}

This study provided a novel algorithm to determine a suitable path interval in multi-axis filleted end milling with a tool inclination. The procedure with wide applicability and robustness was proposed based on the 3D geometrical consideration of filleted end mill. Then, the characteristics of procedure were discussed with the visual data obtained from the computational results. Consequently, the path interval $L / 2$ increased with increasing $R$ in the case that $\rho=5$ and 10 deg. In addition, $L / 2$ rose with increasing $h / R_{c r}$. Especially, the variation of $L / 2$ tended to be a considerable rise in the small range of $h / R_{c r}$. The difference of $L / 2$ between two tools having different $R_{b}$ and $R_{c r}$ was almost 1.5 times in the range from 0 to 0.1 of $h / R_{c r}$. The ratio was equal to the value obtained through dividing the tool with $R=9 \mathrm{~mm}$ by the tool with $R=6 \mathrm{~mm}$. Meanwhile, the computational results showed that $L / 2$ increased with increasing $R_{b}$. Moreover, $L / 2$ increased with increasing $R_{c r}$. From the investigation, the influence of $R_{c r}$ on the variation of $L / 2$ is larger than that of $R_{b}$. These findings would definitely contribute to a path interval determination in multi-axis filleted end milling.

\section{Acknowledgments}

The author would like to thank the financial support provided as the research grant from Faculty of Science and Technology, Seikei University.

\section{REFERENCES}

1. Neugebauer R., Denkena B., Wegener K., Mechatronic systems for machine tools. CIRP Annals Manufacturing Technology, 56, 2007, 657-686.

2. Mohanraja T., Shankarb S., Rajasekarc R., Sakthivela N.R., Pramanikd A., Tool condition monitoring techniques in milling process - a review. Journal of Materials Research and Technology, 2019, DOI: 10.1016/j.jmrt.2019.10.031.
3. Altintas Y., Manufacturing automation metal cutting: mechanics, machine tool vibrations and $\mathrm{CNC}$ design, $2^{\text {nd }}$ edition. Cambridge University Press, 2012.

4. Altintas Y., Brecher C., Weck M., Witt S., Virtual machine tool. CIRP Annals - Manufacturing Technology, 54, 2005, 651-704.

5. Takeuchi Y., CAM for multi-axis control and multitasking machining. Trans. JSME Series C, 77, 2011, 3544-3551 (in Japanese).

6. Konobrytskyi D., Hossain M.M., Tucker T.M., Tarbutton J.A., Kurfess T.R., 5-Axis tool path planning based on highly parallel discrete volumetric geometry representation: Part I. Contact point generation. Computer-AidedDesign and Applications, 15, 2018, 76-89. DOI: 10.1080/16864360.2017.1353730.

7. Lasemi A., Xue D., Gu P., Recent development in CNC machining of freeform surfaces: A stateof-the-art review. Comput. Aided Des., 42, 2010 , 641-654.

8. Bo P., Bartoň M., Plakhotnik D., Pottmann H., Towards efficient 5-axis flank CNC machining of free-form surfaces via fitting envelopes of surfaces of revolution. Comput. Aided Des., 79, 2016, 1-11.

9. Harik R.F., Gong H., Bernard A., 5-axis flank milling: A state-of-the-art review. Comput. Aided Des., 45, 2013, 796-808. DOI: 10.1016/j. cad.2012.08.004.

10. Sekine T., Obikawa T., Normal-unit-vector-based tool path generation using a modified local interpolation for ball-end milling. Journal of Advanced Mechanical Design, Systems, and Manufacturing, 4, 2010, 1246-1260.

11. Bedi S., Ismail F., Mahjoob M.J., Chen Y., Toroidal versus ball nose and flat bottom end mills. International Journal of Advanced Manufacturing Technology, 13, 1997, 326-332.

12. Layegh E., Lazoglu I., 3D surface topography analysis in 5-axis ball-end milling. CIRP Annals, 66, 2017, 133-136.

13. Quinsat Y., Lavernhe S., Lartigue C., Characterization of 3D surface topography in 5-axis milling. Wear, 271, 2011, 590-595.

14. Lee S.G., Kim H.C., Yang M.Y., Mesh-based tool path generation for constant scallop-height machining. International Journal of Advanced Manufacturing Technology, 35, 2008, 15-22.

15. Choi Y.K., Banerjee A., Lee J.W., Tool path generation for free form surfaces using Bézier curves/ surfaces. Computers \& Industrial Engineering, 52, 2007, 486-501.

16. Obikawa T., Sekine T., A higher-order formula of path interval for tool-path generation. International Journal of Automation Technology, 5, 2011, 663-668. 
17. Huang Y., Oliver J.H., Non-constant parameter NC tool path generation on sculptured surfaces. The International Journal of Advanced Manufacturing Technology, 9, 1994, 281-290.

18. Choi B.K., Park J.W., Jun C.S., Cutter-location data optimization in 5-axis surface machining. Computer-Aided Design, 25, 1993, 377-386.

19. Chen T., Shi Z., A tool path generation strategy for three-axis ball-end milling of free-form surfaces. Journal of Materials Processing Technology, 208, 2008, 259-263.

20. Mladenovic G.M., Tanovic L.M., Ehmann K.F., Tool path generation for milling of free form surfaces with feedrate scheduling. FME Transactions, 43, 2015, 9-15.

21. Sarma R., Flat-ended tool swept sections for fiveaxis NC machining of sculptured surfaces. Trans. ASME, Journal of Manufacturing Science and Engineering, 122, 2000, 158-165.

22. Vickers G.W., Quan K.W., Ball-mills versus endmills for curved surface machining. Trans. ASME, Journal of Engineering for Industry, 111, 1989, 22-26.
23. Plakhotnik D., Lauwers B., Computing of the actual shape of removed material for five-axis flatend milling. Computer-Aided Design, 44, 2012, 1103-1114.

24. Lauwers B., Plakhotnik D., Five-axis milling tool path generation with dynamic step-over calculation based on integrated material removal simulation. CIRP Annals - Manufacturing Technology, 61, 2012, 139-142.

25. Kim B.H., Chu C.N., Effect of cutter mark on surface roughness and scallop height in sculptured surface machining. Computer-Aided Design, 26, 1994, 179-188.

26. Sekine T., Obikawa T., Novel path interval determination in 5-axis flat end milling. Applied Mathematical Modelling, 39, 2015, 3459-3480.

27. Sekine T., Obikawa T., Hoshino M., Establishing a novel model for 5-axis milling with filleted end mill. Journal of Advanced Mechanical Design, Systems, and Manufacturing, 6, 2012, 296-309.

28. Sekine T., A 3D geometrical consideration of path interval in filleted end milling. J. Jpn. Soc. Abras. Technol., 60, 515, 2016 (in Japanese). 